EUROPEAN JOURNAL OF PURE AND APPLIED MATHEMATICS

Vol. 12, No. 2, 2019, 553-570

ISSN 1307-5543 - www.ejpam.com

Published by New York Business Global

\title{
On Interval-Valued Fuzzy on Ideal Sets
}

\author{
Mary Joy S. Togonon ${ }^{1}$, Randy L. Caga-anan ${ }^{2, *}$ \\ 1 Bukidnon State University-Baungon Satellite Campus, Bukidnon, Philippines \\ 2 Department of Mathematics and Statistics, College of Science and Mathematics, and \\ Premier Research Institute of Science and Mathematics, MSU-Iligan Institute of Technology, \\ Iligan City, Philippines
}

\begin{abstract}
Fuzzy sets, formalized by Zadeh in 1965, generalizes the classical idea of sets. The idea itself was generalized in 1975 when Zadeh introduced the interval-valued fuzzy sets. In this paper, we generalize further the above concepts by introducing interval-valued fuzzy on ideal sets, where an ideal is a nonempty collection of sets with a property describing the notion of smallness. We develop its basic concepts and properties and consider how one can create mappings of intervalvalued fuzzy on ideal sets from mappings of ordinary sets. We then consider topology and continuity with respect to these sets.
\end{abstract}

2010 Mathematics Subject Classifications: 03E72, 62B86, 94D05

Key Words and Phrases: Fuzzy sets, interval-valued, ideal

\section{Introduction}

In classical set theory, an element either belongs or does not belong to a given set. That is, the membership of elements to a given set is assessed in binary terms. Thus, one may associate a set $A$ on a universal set $U$ to the characteristic function of $A$ with values 0 or 1 . However, there are informations that cannot be precisely assessed as belonging to or not to a given set, like the set of young people in a group. To address this problem, in 1965, Zadeh [9] and Klaua [4] introduced fuzzy sets, where elements have degrees of membership, not just 0 or 1 . Formally defined, a fuzzy set is a mapping from $U$ into the unit interval $[0,1]$. In our example, for a not so young member of the group, a degree of membership equal to 0.2 can be assigned.

In 1978, Zadeh used his theory of fuzzy sets and fuzzy logic to introduce possibility theory [11]. The theory uses a possibility distribution which should not be confused with a probability distribution. Both are fuzzy sets but the sum of the values of a possibility distribution need not be 1 while it should be 1 in a probability distribution. For instance,

${ }^{*}$ Corresponding author.

DOI: https://doi.org/10.29020/nybg.ejpam.v12i2.3418

Email addresses: maryjoy.togonon@g.msuiit.edu.ph (MJ Togonon),

randy.caga-anan@g.msuitt.edu.ph (R. Caga-anan) 
we may assign a value of 0.4 for the possibility that tomorrow there will be rain and a value of 0.7 for the possibility that tomorrow will be sunny. The sum of these two possibilities is already greater than 1 , but our assignment may represent best the information that we know about what will be the weather for tomorrow. Informations like these, with a lot of uncertainties, are not suited to be expressed using a probability distribution.

Now, consider the possibility that tomorrow there will be rain and at the same time it will be sunny. This case is not impossible as it happens rarely in the Philippines. But its possibility should be far less than any of the two separate possibilities. We could not just give it a value equal to the minimum of the two separate possibilities. Expressing information like this motivated the introduction of fuzzy on ideal sets in [6] by Mernilo and Caga-anan. An ideal here is a nonempty collection of subsets of a set $X$, denoted by $\mathcal{I}(X)$, that satisfies:

i. $A \in \mathcal{I}(X)$ and $B \subseteq A$ implies $B \in \mathcal{I}(X)$; and

ii. $A \in \mathcal{I}(X)$ and $B \in \mathcal{I}(X)$ implies $A \cup B \in \mathcal{I}(X)$.

The first property is the reason why an ideal is said to be a collection of sets that are considered small. Ideal spaces were first studied by Kuratowski [5] and Vaidyanathaswamy [8].

Formally, given a nonempty set $X$ and an ideal $\mathcal{I}(X)$ on $X$, a fuzzy on ideal set is a mapping $\mu: \mathcal{I}(X) \rightarrow[0,1]$ such that:

i. $\mu(\varnothing)=0$; and

ii. for nonempty sets $A, B \in \mathcal{I}(X)$, with $A \subseteq B$, we have $\mu(B) \leq \mu(A)$.

The set of all such $\mu$ is denoted by $I^{\mathcal{I}(X)}$. Observe that the reverse inequality $\mu(B) \leq \mu(A)$ encapsulates the preceding idea that the possibility that tomorrow there will be rain and at the same time it will be sunny should not just be equal to the minimum of the separate possibilies as it could be far less. It is also important to note that the preceding definition does not define a measure. For $A \subseteq B$, a measure $m$ should have $m(A) \leq m(B)$, not the reverse inequality, as in our definition. Moreover, any fuzzy set $\alpha$ defined on a set $X$ can be embedded as an element of $I^{\mathcal{P}(X)}$ by associating it with the fuzzy on ideal set $\mu_{\alpha}$ defined by

$$
\mu_{\alpha}(A)= \begin{cases}\alpha(x), & \text { if } A=\{x\}, x \in X \\ 0, & \text { otherwise, }\end{cases}
$$

where $\mathcal{P}(X)$ is the powerset of $X$-the largest ideal of $X$. Thus, fuzzy on ideal sets generalize fuzzy sets.

With regard to uncertainty, there are cases that even the assigning of degrees of membership on a fuzzy set or fuzzy on ideal set has its own uncertainties. In these cases, it is better to give the degree of membership as an interval rather than as a single number. For instance, when one is estimating the age of a person, one has a better chance of capturing the real age by giving a possible range of the age rather than estimating it with a single number. This way, one captures the imprecision better. This lead to the introduction of 
interval-valued fuzzy sets in 1975 by Zadeh [10]. In that same year, it was also considered by Grattan-Guiness [2], Jahn [3] and Sambuc [7].

In this study, we introduce and develop the interval-valued fuzzy on ideal sets. This concept generalizes the above discussed fuzzy sets, fuzzy on ideal sets, and interval-valued fuzzy sets. We formally define it in the next section.

\section{Basic concepts and properties}

Let us first introduce some useful notations. We denote by $\mathscr{I}$ the set of all closed subintervals of $[0,1]$. For $\alpha \in \mathscr{I}$, let $\alpha^{-}$be the left endpoint of $\alpha$ and $\alpha^{+}$be the right endpoint of $\alpha$, so that $\alpha=\left[\alpha^{-}, \alpha^{+}\right]$. Let $\alpha_{1}=\left[\alpha_{1}^{-}, \alpha_{1}^{+}\right]$and $\alpha_{2}=\left[\alpha_{2}^{-}, \alpha_{2}^{+}\right]$be closed subintervals of $\mathscr{I}$. We use the inequality notation " $\leq_{I}$ ", say $\alpha_{1} \leq_{I} \alpha_{2}$, to mean $\alpha_{1}^{-} \leq \alpha_{2}^{-}$ and $\alpha_{1}^{+} \leq \alpha_{2}^{+}$. Let $\mathcal{A}$ be an index set and $\alpha_{i} \in \mathscr{I}$, for each $i \in \mathcal{A}$. We define the supremum of $\alpha_{i}$ by $\sup _{i \in \mathcal{A}} \alpha_{i}=\left[\sup _{i \in \mathcal{A}} \alpha_{i}^{-}, \sup _{i \in \mathcal{A}} \alpha_{i}^{+}\right]$and the infimum of $\alpha_{i}$ by $\inf _{i \in \mathcal{A}} \alpha_{i}=\left[\inf _{i \in \mathcal{A}} \alpha_{i}^{-}, \inf _{i \in \mathcal{A}} \alpha_{i}^{+}\right]$. We define formally an interval-valued fuzzy on ideal set as follows.

Definition 1. Let $X$ be a nonempty set and $\mathcal{I}(X)$ be an ideal on $X$. An interval-valued fuzzy on ideal set (briefly an IVFI set) is a mapping $\widehat{\iota}: \mathcal{I}(X) \rightarrow \mathscr{I}$ that satisfies the following:

i. $\widehat{\iota}(\varnothing)=[0,0] ;$ and

ii. for nonempty sets $A, B \in \mathcal{I}(X)$ with $A \subseteq B$,

$$
\widehat{\iota}(B) \leq_{I} \widehat{\iota}(A) .
$$

We denote the set of all such $\widehat{\iota}$ by $\mathscr{I}^{\mathcal{I}(X)}$.

Remark 1. In a similar way that fuzzy on ideal sets generalize fuzzy sets, as discussed above, IVFI sets generalize interval-valued fuzzy sets.

Example 1. Let $X$ be a nonempty set and $\pi: X \rightarrow \mathscr{I}$ be an interval-valued fuzzy set. We can define an IVFI set $\widehat{\pi}: \mathcal{P}(X) \rightarrow \mathscr{I}$ by

$$
\widehat{\pi}(A)= \begin{cases}{[0,0],} & \text { if } A=\varnothing ; \\ \inf _{x \in A} \pi(x), & \text { if } A \neq \varnothing, A \in \mathcal{P}(X) .\end{cases}
$$

This is similar to the guaranteed possibility given in [1].

We call an IVFI set $\widehat{\iota}: \mathcal{I}(X) \rightarrow \mathscr{I}$ with the property that for all nonempty set $A \in \mathcal{I}(X), \widehat{\iota}(A)=\inf _{x \in A} \widehat{\iota}(\{x\})$, a guaranteed possibility IVFI set.

Remark 2. Let $X$ be a nonempty set and $\mathcal{I}(X)$ be an ideal on $X$. We denote by $\widetilde{0}_{\mathcal{I}(X)}$ the IVFI set $\widetilde{0}_{\mathcal{I}(X)}: \mathcal{I}(X) \rightarrow\{[0,0]\}$ and by $\widetilde{1}_{\mathcal{I}(X)}$ the IVFI set

$$
\widetilde{1}_{\mathcal{I}(X)}(A)= \begin{cases}{[0,0],} & \text { if } A=\varnothing ; \\ {[1,1],} & \text { if } A \neq \varnothing, A \in \mathcal{I}(X) .\end{cases}
$$


Next, we define some relational operators between IVFI sets.

Definition 2. Let $X$ be a nonempty set and $\mathcal{I}(X)$ be an ideal on $X$. Let $\widehat{\iota} \widehat{\tau} \in \mathscr{I}^{\mathcal{I}(X)}$. We say

(i) $\widehat{\iota}$ is a subset of $\widehat{\tau}$, denoted by $\widehat{\iota} \leqslant \widehat{\tau}$, if $\widehat{\iota}(A) \leq_{I} \widehat{\tau}(A)$, for all $A \in \mathcal{I}(X)$; and

(ii) $\widehat{\iota}$ is equal to $\widehat{\tau}$, denoted by $\widehat{\iota}=\widehat{\tau}$, if $\widehat{\iota}(A)=\widehat{\tau}(A)$, for all $A \in \mathcal{I}(X)$.

Remark 3. To avoid confusion, we summarize first our "inequality" notations.

$i$. The symbol " $\leq$ " for the usual inequality with the real numbers.

ii. The symbol " $\leq_{I}$ " for the inequality with intervals.

iii. The symbol " $\leqslant$ " to denote the subset relation with IVFI sets.

Next, we define complement, union, and intersection of IVFI sets. We then prove that the resulting mappings are also IVFI sets, showing that our definitions are well-defined.

Definition 3. Let $X$ be a nonempty set and $\mathcal{I}(X)$ be an ideal on $X$. Let $\widehat{\iota} \in \mathscr{I}^{\mathcal{I}(X)}$. The complement of $\widehat{\iota}$, denoted by $\widehat{\iota}^{c}$, is defined by, $\widehat{\iota}^{c}(\varnothing)=[0,0]$ and for every nonempty set $A \in \mathcal{I}(X)$,

$$
\widehat{\iota}^{c}(A)=\left[\inf _{x \in A}\left\{1-[\widehat{\iota}(\{x\})]^{+}\right\}, \inf _{x \in A}\left\{1-[\widehat{\iota}(\{x\})]^{-}\right\}\right] .
$$

Proposition 1. Let $X$ be a nonempty set and $\mathcal{I}(X)$ be an ideal on $X$. If $\widehat{\iota} \in \mathscr{I}^{\mathcal{I}(X)}$, then the complement of $\widehat{\iota}$ is an IVFI set.

Proof. Let $\widehat{\iota} \in \mathscr{I}^{\mathcal{I}(X)}$. Let $A \in \mathcal{I}(X), x \in A$ and $\widehat{\iota}(\{x\})=\left[[\widetilde{\iota}(\{x\})]^{-},[\widehat{\iota}(\{x\})]^{+}\right]$. Since $[\widehat{\iota}(\{x\})]^{-} \leq[\widehat{\iota}(\{x\})]^{+}$, we have $1-[\widehat{\iota}(\{x\})]^{+} \leq 1-[\widehat{\iota}(\{x\})]^{-}$. Hence,

$$
\inf _{x \in A}\left\{1-[\widehat{\iota}(\{x\})]^{+}\right\} \leq \inf _{x \in A}\left\{1-[\widehat{\iota}(\{x\})]^{-}\right\},
$$

and indeed we have a closed interval. We need to show that the reverse inequality of an IVFI set holds. Let $\varnothing \neq A, B \in \mathcal{I}(X)$ such that $A \subseteq B$. Then, $\left\{1-[\widehat{\iota}(\{x\})]^{-}: x \in A\right\} \subseteq$ $\left\{1-[\widehat{\iota}(\{x\})]^{-}: x \in B\right\}$ and $\left\{1-[\widehat{\iota}(\{x\})]^{+}: x \in A\right\} \subseteq\left\{1-[\widehat{\iota}(\{x\})]^{+}: x \in B\right\}$. Hence,

$$
\begin{aligned}
\widehat{\iota}^{c}(B) & =\left[\inf _{x \in B}\left\{1-[\widehat{\iota}(\{x\})]^{+}\right\}, \inf _{x \in B}\left\{1-[\widehat{\iota}(\{x\})]^{-}\right\}\right] \\
& \leq_{I}\left[\inf _{x \in A}\left\{1-[\widehat{\iota}(\{x\})]^{+}\right\}, \inf _{x \in A}\left\{1-[\widehat{\iota}(\{x\})]^{-}\right\}\right] \\
& =\widehat{\iota}^{c}(A) .
\end{aligned}
$$

Therefore, $\widehat{\iota}^{c}$ is an IVFI set.

Remark 4. For a singleton set $A=\{x\} \in \mathcal{I}(X)$, the preceding definition coincides with the definition of the complement of an interval-valued fuzzy set. 
Definition 4. Let $X$ be a nonempty set and $\mathcal{I}(X)$ be an ideal on $X$. Let $\widehat{\iota}, \widehat{\tau} \in \mathscr{I} \mathcal{I}(X)$. The union and intersection of $\widehat{\iota}$ and $\widehat{\tau}$, denoted by $\widehat{\iota} \widehat{\tau}$ and $\widehat{\iota} \wedge \widehat{\tau}$, respectively, are given by

$$
(\widehat{\iota} \vee \widehat{\tau})(A)=\left[\max \left\{[\widehat{\iota}(A)]^{-},[\widehat{\tau}(A)]^{-}\right\}, \max \left\{[\widehat{\iota}(A)]^{+},[\widehat{\tau}(A)]^{+}\right\}\right]
$$

and

$$
(\widehat{\iota} \wedge \widehat{\tau})(A)=\left[\min \left\{[\widehat{\iota}(A)]^{-},[\widehat{\tau}(A)]^{-}\right\}, \min \left\{[\widehat{\iota}(A)]^{+},[\widehat{\tau}(A)]^{+}\right\}\right],
$$

for all $A \in \mathcal{I}(X)$, respectively.

In general, the union and intersection of a collection of IVFI sets $\left\{\widehat{\iota}_{j}: j \in J\right\}$, denoted by $\bigvee_{j \in J} \widehat{\iota}_{j}$ and $\bigwedge_{j \in J} \widehat{\iota}_{j}$, are given by

$$
\left(\bigvee_{j \in J} \widehat{\iota}_{j}\right)(A)=\left[\sup \left\{\left[\widehat{\iota}_{j}(A)\right]^{-}: j \in J\right\}, \sup \left\{\left[\widehat{\iota}_{j}(A)\right]^{+}: j \in J\right\}\right]
$$

and

$$
\left(\bigwedge_{j \in J} \widehat{\iota}_{j}\right)(A)=\left[\inf \left\{\left[\widehat{\iota}_{j}(A)\right]^{-}: j \in J\right\}, \inf \left\{\left[\widehat{\iota}_{j}(A)\right]^{+}: j \in J\right\}\right],
$$

for all $A \in \mathcal{I}(X)$, respectively.

One can easily check that the arbitrary union or intersection of IVFI sets is an IVFI set.

The following are some properties of the operations on IVFI sets.

Theorem 1. Let $X$ be a nonempty set and $\mathcal{I}(X)$ be an ideal on $X$. Let $\widehat{\iota}, \widehat{\tau}, \widehat{\eta} \in \mathscr{I}^{\mathcal{I}(X)}$. Then,

i. (Commutativity): $\widehat{\iota} \vee \widehat{\tau}=\widehat{\tau} \vee \widehat{\iota}$ and $\widehat{\iota} \wedge \widehat{\tau}=\widehat{\tau} \wedge \widehat{\iota}$.

ii. (Associativity): $(\widehat{\iota} \vee \widehat{\tau}) \vee \widehat{\eta}=\widehat{\iota} \vee(\widehat{\tau} \vee \widehat{\eta})$ and $(\widehat{\iota} \wedge \widehat{\tau}) \wedge \widehat{\eta}=\widehat{\iota} \wedge(\widehat{\tau} \wedge \widehat{\eta})$.

iii. (Transitivity): If $\widehat{\iota} \leqslant \widehat{\tau}$ and $\widehat{\tau} \leqslant \widehat{\eta}$, then $\widehat{\iota} \leqslant \widehat{\eta}$.

iv. (Distributivity): $\widehat{\iota} \vee(\widehat{\tau} \wedge \widehat{\eta})=(\widehat{\iota} \vee \widehat{\tau}) \wedge(\widehat{\iota} \vee \widehat{\eta})$ and $\widehat{\iota} \wedge(\widehat{\tau} \vee \widehat{\eta})=(\widehat{\iota} \wedge \widehat{\tau}) \vee(\widehat{\iota} \wedge \widehat{\eta})$

v. (De Morgan's Law): $(\widehat{\iota} \vee \widehat{\tau})^{c}=\widehat{\iota}^{c} \wedge \widehat{\tau}^{c}$ and $(\widehat{\iota} \wedge \widehat{\tau})^{c}=\widehat{\iota}^{c} \vee \widehat{\tau}^{c}$

Proof. Let $\widehat{\iota}, \widehat{\tau}, \widehat{\eta} \in \mathscr{I}^{\mathcal{I}(X)}$. Properties $(i)$ and $(i i)$ follows from the commutativity and associativity of the maximum and minimum operations. Suppose that $\widehat{\iota} \leqslant \widehat{\tau}$ and $\widehat{\tau} \leqslant \widehat{\eta}$. Then for all $A \in \mathcal{I}(X), \widehat{\iota}(A) \leq_{I} \widehat{\tau}(A)$ and $\widehat{\tau}(A) \leq_{I} \widehat{\eta}(A)$, which implies that $\widehat{\iota}(A) \leq_{I} \widehat{\eta}(A)$, for all $A \in \mathcal{I}(X)$. That is, $\widehat{\iota} \leqslant \widehat{\eta}$, easily proving (iii). To prove $(i v)$, let $A \in \mathcal{I}(X)$ and consider that

$$
\begin{aligned}
(\widehat{\iota} \vee(\widehat{\tau} \wedge \widehat{\eta}))(A) & =\left[\max \left\{[\widehat{\iota}(A)]^{-},[(\widehat{\tau} \wedge \widehat{\eta})(A)]^{-}\right\}, \max \left\{[\widehat{\iota}(A)]^{+},[(\widehat{\tau} \wedge \widehat{\eta})(A)]^{+}\right\}\right] \\
& =\left[\max \left\{[\widehat{\iota}(A)]^{-}, \min \left\{[\widehat{\tau}(A)]^{-},[\widehat{\eta}(A)]^{-}\right\}\right\}, \max \left\{[\widehat{\iota}(A)]^{+}, \min \left\{[\widehat{\tau}(A)]^{+},[\widehat{\eta}(A)]^{+}\right\}\right\}\right] .
\end{aligned}
$$


We consider two cases. First, if $\widehat{\tau}(A) \leq_{I} \widehat{\eta}(A)$, then

$$
(\widehat{\iota} \vee(\widehat{\tau} \wedge \widehat{\eta}))(A)=\left[\max \left\{[\widehat{\iota}(A)]^{-},[\widehat{\tau}(A)]^{-}\right\}, \max \left\{[\widehat{\iota}(A)]^{+},[\widehat{\tau}(A)]^{+}\right\}\right] .
$$

Second, if $\widehat{\eta}(A) \leq_{I} \widehat{\tau}(A)$, then

$$
(\widehat{\iota} \vee(\widehat{\tau} \wedge \widehat{\eta}))(A)=\left[\max \left\{[\widehat{\iota}(A)]^{-},[\widehat{\eta}(A)]^{-}\right\}, \max \left\{[\widehat{\iota}(A)]^{+},[\widehat{\eta}(A)]^{+}\right\}\right] .
$$

Summarizing the two cases, we have

$$
\begin{aligned}
(\widehat{\iota} \vee(\widehat{\tau} \wedge \widehat{\eta}))(A)= & {\left[\min \left\{\max \left\{[\widehat{\iota}(A)]^{-},[\widehat{\tau}(A)]^{-}\right\}, \max \left\{[\widehat{\iota}(A)]^{-},[\widehat{\eta}(A)]^{-}\right\}\right\},\right.} \\
& \left.\min \left\{\max \left\{[\widehat{\iota}(A)]^{+},[\widehat{\tau}(A)]^{+}\right\}, \max \left\{[\widehat{\iota}(A)]^{+},[\widehat{\eta}(A)]^{+}\right\}\right\}\right] \\
= & ((\widehat{\iota} \vee \widehat{\tau}) \wedge(\widehat{\iota} \vee \widehat{\eta}))(A) .
\end{aligned}
$$

Thus, $\widehat{\iota}(\widehat{\tau} \wedge \widehat{\eta})=(\widehat{\iota} \vee \widehat{\tau}) \wedge(\widehat{\iota} \vee \widehat{\eta})$. Similarly, we can show that $\widehat{\iota} \wedge(\widehat{\tau} \vee \widehat{\eta})=(\widehat{\iota} \wedge \widehat{\tau}) \vee(\widehat{\iota} \wedge \widehat{\eta})$. To prove $(v)$, let $\varnothing \neq A \in \mathcal{I}(X)$ and note that

$$
\begin{aligned}
(\widehat{\iota} \vee \widehat{\tau})^{c}(A) & =\left[\inf _{x \in A}\left\{1-[(\widehat{\iota} \vee \widehat{\tau})(\{x\})]^{+}\right\}, \inf _{x \in A}\left\{1-[(\widehat{\iota} \vee \widehat{\tau})(\{x\})]^{-}\right\}\right] \\
& =\left[\inf _{x \in A}\left\{1-\max \left\{[\widehat{\iota}(\{x\})]^{+},[\widehat{\tau}(\{x\})]^{+}\right\}\right\}, \inf _{x \in A}\left\{1-\max \left\{[\widehat{\iota}(\{x\})]^{-},[\widehat{\tau}(\{x\})]^{-}\right\}\right\}\right] .
\end{aligned}
$$

We also consider two cases. First, if $\widehat{\tau}(\{x\}) \leq_{I} \widehat{\iota}(\{x\})$, then

$$
(\widehat{\iota} \vee \widehat{\tau})^{c}(A)=\left[\inf _{x \in A}\left\{1-[\widehat{\iota}(\{x\})]^{+}\right\}, \inf _{x \in A}\left\{1-[\widehat{\iota}(\{x\})]^{-}\right\}\right] .
$$

Second, if $\widehat{\iota}(\{x\}) \leq_{I} \widehat{\tau}(\{x\})$, then

$$
(\widehat{\iota} \vee \widehat{\tau})^{c}(A)=\left[\inf _{x \in A}\left\{1-[\widehat{\tau}(\{x\})]^{+}\right\}, \inf _{x \in A}\left\{1-[\widehat{\tau}(\{x\})]^{-}\right\}\right] .
$$

Combining the two cases, we have

$$
\begin{aligned}
(\widehat{\iota} \vee \widehat{\tau})^{c}(A)= & {\left[\min \left\{\inf _{x \in A}\left\{1-[\widehat{\iota}(\{x\})]^{+}\right\}, \inf _{x \in A}\left\{1-[\widehat{\tau}(\{x\})]^{+}\right\}\right\},\right.} \\
& \left.\min \left\{\inf _{x \in A}\left\{1-[\widehat{\iota}(\{x\})]^{-}\right\}, \inf _{x \in A}\left\{1-[\widehat{\tau}(\{x\})]^{-}\right\}\right\}\right] \\
= & \left(\widehat{\iota}^{c} \wedge \widehat{\tau}^{c}\right)(A) .
\end{aligned}
$$

Hence, $(\widehat{\iota} \widehat{\tau})^{c}=\widehat{\iota}^{c} \wedge \widehat{\tau}^{c}$. Using the same argument, we can also show that $(\widehat{\iota} \wedge \widehat{\tau})^{c}=\widehat{\iota}^{c} \vee \widehat{\tau}^{c}$, and our proof is complete.

The next theorem states some interesting properties of the complement of IVFI sets.

Theorem 2. Let $X$ be a nonempty set and $\mathcal{I}(X)$ be an ideal on $X$. Let $\widehat{\iota}, \widehat{\tau} \in \mathscr{I}^{\mathcal{I}(X)}$. Then,

i. $\widehat{\iota} \leqslant\left(\widehat{\iota}^{c}\right)^{c}$ 
ii. $\widehat{\iota}=\left(\widehat{\iota}^{c}\right)^{c}$ if and only if $\widehat{\iota}$ is a guaranteed possibility IVFI set; and

iii. if $\widehat{\iota} \leqslant \widehat{\tau}$, then $\widehat{\tau}^{c} \leqslant \widehat{\iota}^{c}$.

Proof. Let $\widehat{\iota} \in \mathscr{I}^{\mathcal{I}(X)}$ and $\varnothing \neq A \in \mathcal{I}(X)$. Note first that for singleton sets $\{x\} \in \mathcal{I}(X)$, we have $\widehat{\iota}^{c}(\{x\})=\left[1-[\widehat{\iota}(\{x\})]^{+}, 1-[\widehat{\iota}(\{x\})]^{-}\right]$. Then, consider that

$$
\begin{aligned}
\left(\widehat{\iota}^{c}\right)^{c}(A) & =\left[\inf _{x \in A}\left\{1-\left[\widetilde{\iota}^{c}(\{x\})\right]^{+}\right\}, \inf _{x \in A}\left\{1-\left[\widehat{\iota}^{c}(\{x\})\right]^{-}\right\}\right] \\
& =\left[\inf _{x \in A}\left\{1-\left(1-[\widehat{\iota}(\{x\})]^{-}\right)\right\}, \inf _{x \in A}\left\{1-\left(1-\widehat{\iota}[(\{x\})]^{+}\right)\right\}\right] \\
& =\left[\inf _{x \in A}[\widehat{\iota}(\{x\})]^{-}, \inf _{x \in A}[\widehat{\iota}(\{x\})]^{+}\right] .
\end{aligned}
$$

Since $\widehat{\iota}$ is an IVFI set, the reverse inequality property implies that

$$
\widehat{\iota}(A)=\left[[\widehat{\iota}(A)]^{-},[\widehat{\iota}(A)]^{+}\right] \leq_{I}\left[\inf _{x \in A}[\widehat{\iota}(\{x\})]^{-}, \inf _{x \in A}[\widehat{\iota}(\{x\})]^{+}\right] .
$$

Thus, $\widehat{\iota} \leqslant\left(\widehat{\iota}^{c}\right)^{c}$, proving $(i)$. To prove $(i i)$, recall first the definition of a guaranteed possibility IVFI set after Example 1. Now, suppose that $\widehat{\iota}=\left(\widehat{\iota}^{c}\right)^{c}$. Then, $\widehat{\iota}(A)=\left(\widehat{\iota}^{c}\right)^{c}(A)$, for all $A \in \mathcal{I}(X)$. Note from (1) that for $\varnothing \neq A \in \mathcal{I}(X)$,

$$
\left(\widehat{\iota}^{c}\right)^{c}(A)=\left[\inf _{x \in A}[\widehat{\iota}(\{x\})]^{-}, \inf _{x \in A}[\widehat{\iota}(\{x\})]^{+}\right] .
$$

Thus, $\widehat{\iota}(A)=\left[\inf _{x \in A}[\widehat{\iota}(\{x\})]^{-}, \inf _{x \in A}[\widehat{\iota}(\{x\})]^{+}\right]=\inf _{x \in A} \widehat{\iota}(\{x\})$. That is, $\widehat{\iota}$ is a guaranteed possibility IVFI set. Conversely, suppose that $\widehat{\iota}(A)=\left[\inf _{x \in A} \widehat{\iota}(\{x\})^{-}, \inf _{x \in A} \widehat{\iota}(\{x\})^{+}\right]$, for $\varnothing \neq A \in \mathcal{I}(X)$. Then by $(1), \widehat{\iota}(A)=\left(\widehat{\iota}^{c}\right)^{c}(A)$. Thus, $\widehat{\iota}=\left(\widehat{\iota}^{c}\right)^{c}$. To prove (iii), suppose that $\widehat{\iota} \leqslant \widehat{\tau}$. Let $\varnothing \neq A \in \mathcal{I}(X)$. Then, $\widehat{\iota}(\{x\}) \leq_{I} \widehat{\tau}(\{x\})$, for all $x \in A$. Thus, for every $x \in A$,

$$
\left[1-[\widehat{\tau}(\{x\})]^{+}, 1-[\widehat{\tau}(\{x\})]^{-}\right] \leq_{I}\left[1-[\widehat{\iota}(\{x\})]^{+}, 1-[\widehat{\iota}(\{x\})]^{-}\right] .
$$

Hence,

$\left[\inf _{x \in A}\left\{1-[\widehat{\tau}(\{x\})]^{+}\right\}, \inf _{x \in A}\left\{1-[\widehat{\tau}(\{x\})]^{-}\right\}\right] \leq_{I}\left[\inf _{x \in A}\left\{1-[\widehat{\iota}(\{x\})]^{+}\right\}, \inf _{x \in A}\left\{1-[\widehat{\iota}(\{x\})]^{-}\right\}\right]$.

Thus, $\widehat{\tau}^{c}(A) \leq_{I} \widehat{\iota}^{c}(A)$, for all $A \in \mathcal{I}(X)$. Therefore, $\widehat{\tau}^{c} \leqslant \widehat{\iota}^{c}$.

\section{Mappings}

Let $X$ and $Y$ be nonempty sets and $f: X \rightarrow Y$ be a mapping. Moreover, let $\mathcal{I}(X)$ and $\mathcal{I}(Y)$ be ideals on $X$ and $Y$, respectively. We define the image and pre-image of the ideals under $f$ by $f(\mathcal{I}(X))=\{f(A): A \in \mathcal{I}(X)\}$ and $f^{-1}(\mathcal{I}(Y))=\left\{A: A \subseteq f^{-1}(B), B \in\right.$ $\mathcal{I}(Y)\}$, where $f(A)$ and $f^{-1}(B)$ is the usual image and preimage of $A \subseteq X$ and $B \subseteq Y$, respectively. The next theorem is important because it shows that these image and preimage of ideals are also ideals. The proof can be found in [6]. 
Theorem $3([6])$. Let $X$ and $Y$ be nonempty sets and let $f: X \rightarrow Y$ be a mapping. If $\mathcal{I}(X)$ and $\mathcal{I}(Y)$ are ideals on $X$ and $Y$, respectively, then $f(\mathcal{I}(X))$ and $f^{-1}(\mathcal{I}(Y))$ are ideals on $Y$ and $X$, respectively.

Given a mapping of two ordinary sets, we define the image and pre-image of IVFI sets. We then prove that these image and pre-image are also IVFI sets, showing that they are well-defined.

Definition 5. Let $X$ and $Y$ be nonempty sets and $f: X \rightarrow Y$ be a mapping. Moreover, let $\mathcal{I}(X)$ and $\mathcal{I}(Y)$ be ideals on $X$ and $Y$, respectively.

i. If $\widehat{\iota} \in \mathscr{I}^{\mathcal{I}(X)}$, then the image of $\widehat{\iota}$ under $f$, denoted by $f[\widehat{\iota}]$, is the mapping $f[\hat{\iota}]$ : $f(\mathcal{I}(X)) \rightarrow \mathscr{I}$ given by

$$
(f[\widehat{\iota}])(B)=\left[\sup _{A \in S}[\widehat{\iota}(A)]^{-}, \sup _{A \in S}[\widehat{\iota}(A)]^{+}\right],
$$

where $S=\{A \in \mathcal{I}(X): f(A)=B\}$.

ii. If $\widehat{\tau} \in \mathscr{I}^{\mathcal{I}(Y)}$, then the pre-image of $\widehat{\tau}$ under $f$, denoted by $f^{-1}[\widehat{\tau}]$, is the mapping $f^{-1}[\widehat{\tau}]: f^{-1}(\mathcal{I}(Y)) \rightarrow \mathscr{I}$ given by

$$
\left(f^{-1}[\widehat{\tau}]\right)(A)=(\widehat{\tau} \circ f)(A),
$$

where $(\widehat{\tau} \circ f)(A)$ is the composition $\widehat{\tau}(f(A))$.

Let $B \in f(\mathcal{I}(X))$ and $S=\{A \in \mathcal{I}(X): f(A)=B\}$. If $B=\varnothing$, then $S=\{\varnothing\}$, and so $\sup _{A \in S} \widehat{\iota}(A)=[0,0]$. Also, if $\varnothing=A \in f^{-1}(\mathcal{I}(Y))$, then $f(A)=\varnothing$ and so $\widehat{\tau}(f(A))=[0,0]$. Hence, we have the following remark.

Remark 5. Let $X$ and $Y$ be nonempty sets and $f: X \rightarrow Y$ be a mapping. Let $\widehat{\iota}$ and $\widehat{\tau}$ be IVFI sets in $\mathscr{I}^{\mathcal{I}(X)}$ and $\mathscr{I}^{\mathcal{I}(Y)}$, respectively. Then, we have $f[\widehat{\iota}](\varnothing)=[0,0]$ and $f^{-1}[\widehat{\tau}](\varnothing)=[0,0]$.

Theorem 4. Let $X$ and $Y$ be nonempty sets and $f: X \rightarrow Y$ be a mapping. Let $\widehat{\iota}$ and $\widehat{\tau}$ be IVFI sets defined on the ideals $\mathcal{I}(X)$ and $\mathcal{I}(Y)$, respectively. Then, $f[\widehat{\imath}]$ and $f^{-1}[\widehat{\tau}]$ are IVFI sets defined on the ideals $f(\mathcal{I}(X))$ and $f^{-1}(\mathcal{I}(Y))$, respectively.

Proof. We first show that $f[\hat{\imath}]$ is an IVFI set defined on the ideal $f(\mathcal{I}(X))$. Let $\varnothing \neq B_{1}, B_{2} \in f(\mathcal{I}(X))$ such that $B_{1} \subseteq B_{2}$. Let $S_{1}=\left\{A \in \mathcal{I}(X): f(A)=B_{1}\right\}$ and $S_{2}=\left\{A \in \mathcal{I}(X): f(A)=B_{2}\right\}$. Since $\mathcal{I}(X)$ is an ideal, for every $A \in \mathcal{I}(X)$ such that $f(A)=B_{2}$, there exists $A_{1} \in \mathcal{I}(X)$ such that $A_{1} \subseteq A$ and $f\left(A_{1}\right)=B_{1}$. Since $\widehat{\iota}$ is an IVFI set and $A_{1} \subseteq A$, we have $\widehat{\iota}(A) \leq_{I} \widehat{\iota}\left(A_{1}\right)$. Hence, $\sup _{A \in S_{2}} \widehat{\iota}(A) \leq_{I} \sup _{A_{1} \in S_{1}} \widehat{\iota}\left(A_{1}\right)$. Thus, $f[\widehat{\iota}]\left(B_{2}\right) \leq_{I} f[\widehat{\imath}]\left(B_{1}\right)$. Therefore, with Remark 5 and Theorem $3, f[\widehat{\imath}]$ is an IVFI set defined on the ideal $f(\mathcal{I}(X))$. Next, to show that $f^{-1}[\widehat{\tau}]$ is an IVFI set defined on the ideal $f^{-1}(\mathcal{I}(Y))$, let $A, A_{1} \in f^{-1}(\mathcal{I}(Y))$ such that $A_{1} \subseteq A$. Then, $f\left(A_{1}\right) \subseteq f(A)$ and $f\left(A_{1}\right), f(A) \in \mathcal{I}(Y)$. Since $\widehat{\tau}$ is an IVFI set on $\mathcal{I}(Y), \widehat{\tau}(f(A)) \leq_{I} \widehat{\tau}\left(f\left(A_{1}\right)\right)$. Thus, 
$f^{-1}[\widehat{\tau}](A) \leq_{I} f^{-1}[\widehat{\tau}]\left(A_{1}\right)$. Therefore, with Remark 5 and Theorem $3, f^{-1}[\widehat{\tau}]$ is an IVFI set defined on the ideal $f^{-1}(\mathcal{I}(Y))$.

We can extend our result to composition of mappings. The following corollaries are immediate consequences of Theorem 3 and Theorem 5 .

Corollary 1. Let $X, Y$, and $Z$ be nonempty sets and $f: X \rightarrow Y$ and $g: Y \rightarrow Z$ be mappings. Let $g \circ f: X \rightarrow Z$ be a composition map. If $\mathcal{I}(X)$ and $\mathcal{I}(Z)$ are ideals on $X$ and $Z$, respectively, then, $(g \circ f)(\mathcal{I}(X))=g(f(\mathcal{I}(X)))$ and $(g \circ f)^{-1}(\mathcal{I}(Z))=f^{-1}\left(g^{-1}(\mathcal{I}(Z))\right)$ are ideals on $Z$ and $X$, respectively.

Corollary 2. Let $X, Y$, and $Z$ be nonempty sets and $f: X \rightarrow Y$ and $g: Y \rightarrow Z$ be mappings. Let $g \circ f: X \rightarrow Z$ be a composition map and, $\mathcal{I}(X)$ and $\mathcal{I}(Z)$ are ideals on $X$ and $Z$, respectively. If $\widehat{\iota} \in \mathscr{I}^{\mathcal{I}(X)}$ and $\widehat{\eta} \in \mathscr{I}^{\mathcal{I}(Z)}$, then $(g \circ f)[\hat{\imath}]$ and $(g \circ f)^{-1}(\widehat{\eta})$ are IVFI sets defined on $(g \circ f)(\mathcal{I}(X))$ and $(g \circ f)^{-1}(\mathcal{I}(Z))$, respectively.

The next theorem state some properties of the defined mappings of IVFI sets. We start with the following needed proposition.

Proposition 2. Let $X$ and $Y$ be nonempty sets and, $\mathcal{I}(X)$ and $\mathcal{I}(Y)$ be ideals in $X$ and $Y$, respectively. Let $f: X \rightarrow Y$ be a mapping. Then,

i. $f\left(f^{-1}(\mathcal{I}(Y))\right)=\mathcal{I}(Y)$, if $f$ is onto; and

ii. $f^{-1}(f(\mathcal{I}(X)))=\mathcal{I}(X)$, if $f$ is one-to-one.

Proof. Suppose that $f$ is onto. Let $B \in f\left(f^{-1}(\mathcal{I}(Y))\right)$. Then there exists $A \in$ $f^{-1}(\mathcal{I}(Y))$ such that $f(A)=B$. Since $A \in f^{-1}(\mathcal{I}(Y)), A \subseteq f^{-1}\left(B_{1}\right)$ for some $B_{1} \in \mathcal{I}(Y)$. Note that $f(A) \subseteq f\left(f^{-1}\left(B_{1}\right)\right)=B_{1}$, since $f$ is onto. Thus, $B \subseteq B_{1}$. By the definition of an ideal, $B \in \mathcal{I}(Y)$. Hence, $f\left(f^{-1}(\mathcal{I}(Y))\right) \subseteq \mathcal{I}(Y)$. Conversely, let $B \in \mathcal{I}(Y)$ and $C=f^{-1}(B)$. Then $C \in f^{-1}(\mathcal{I}(Y))$. We thus have $f(C) \in f\left(f^{-1}(\mathcal{I}(Y))\right)$. Since $f$ is onto, $B=f\left(f^{-1}(B)\right)=f(C) \in f\left(f^{-1}(\mathcal{I}(Y))\right)$. Thus, $\mathcal{I}(Y) \subseteq f\left(f^{-1}(\mathcal{I}(Y))\right)$. Therefore, $f\left(f^{-1}(\mathcal{I}(Y))\right)=\mathcal{I}(Y)$.

Suppose that $f$ is one-to-one. Let $A \in f^{-1}(f(\mathcal{I}(X)))$. Then there exists $B \in f(\mathcal{I}(X))$ such that $f^{-1}(B)=A$. Since $B \in f(\mathcal{I}(X)), B=f\left(A_{1}\right)$ for some $A_{1} \in \mathcal{I}(X)$. Since $f$ is one-to-one, we have $A=f^{-1}(B)=f^{-1}\left(f\left(A_{1}\right)\right)=A_{1}$. Thus, $A \in \mathcal{I}(X)$. Hence, $f^{-1}(f(\mathcal{I}(X))) \subseteq \mathcal{I}(X)$. Conversely, let $A \in \mathcal{I}(X)$ and $D=f(A)$. Then, $D \in f(\mathcal{I}(X))$. We thus have $f^{-1}(D) \in f^{-1}(f(\mathcal{I}(X)))$. Since $f$ is one-to-one, $A=f^{-1}(f(A))=f^{-1}(D) \in$ $f^{-1}(f(\mathcal{I}(X)))$. Thus, $\mathcal{I}(X) \subseteq f^{-1}(f(\mathcal{I}(X)))$. Therefore, $f^{-1}(f(\mathcal{I}(X)))=\mathcal{I}(X)$.

Theorem 5. Let $X$ and $Y$ be nonempty sets and, $\mathcal{I}(X)$ and $\mathcal{I}(Y)$ be ideals in $X$ and $Y$, respectively. Let $f: X \rightarrow Y$ be a mapping. If $\widehat{\iota}, \widehat{\tau} \in \mathscr{I}^{\mathcal{I}(X)}$ and $\widehat{\omega}, \widehat{\eta} \in \mathscr{I}^{\mathcal{I}(Y)}$, then

i. $f^{-1}\left[\widehat{\eta}^{c}\right]=\left(f^{-1}[\widehat{\eta}]\right)^{c}$;

ii. $(f[\widehat{\iota}])^{c} \leqslant f\left[\widehat{\iota}^{c}\right]$ 
iii. if $\widehat{\omega} \leqslant \widehat{\eta}$, then $f^{-1}[\widehat{\omega}] \leqslant f^{-1}[\widehat{\eta}]$;

iv. if $\widehat{\iota} \leqslant \widehat{\tau}$, then $f[\hat{\iota}] \leqslant f[\widehat{\tau}]$;

v. if $f$ is onto, then $f\left[f^{-1}[\widehat{\eta}]\right]=\widehat{\eta}$; and

vi. if $f$ is one-to-one, then $\widehat{\iota} \leqslant f^{-1}[f[\widehat{\iota}]]$.

\section{Proof.}

i. Let $\varnothing \neq A \in f^{-1}(\mathcal{I}(Y))$. Then

$$
\begin{aligned}
\left(f^{-1}[\widehat{\eta}]\right)^{c}(A) & =\left[\inf _{x \in A}\left\{1-\left[\left(f^{-1}[\widehat{\eta}]\right)(\{x\})\right]^{+}\right\}, \inf _{x \in A}\left\{1-\left[\left(f^{-1}[\widehat{\eta}]\right)(\{x\})\right]^{-}\right\}\right] \\
& =\left[\inf _{x \in A}\left\{1-[(\widehat{\eta} \circ f)(\{x\})]^{+}\right\}, \inf _{x \in A}\left\{1-[(\widehat{\eta} \circ f)(\{x\})]^{-}\right\}\right] \\
& =\left[\inf _{x \in A}\left\{1-[\widehat{\eta}(f(\{x\}))]^{+}\right\}, \inf _{x \in A}\left\{1-[\widehat{\eta}(f(\{x\}))]^{-}\right\}\right] \\
& =\left[\inf _{f(x) \in f(A)}\left\{1-[\widehat{\eta}(f(\{x\}))]^{+}\right\}, \inf _{f(x) \in f(A)}\left\{1-[\widehat{\eta}(f(\{x\}))]^{-}\right\}\right] \\
& =\left[\left[\widehat{\eta}^{c}(f(A))\right]^{-},\left[\widehat{\eta}^{c}(f(A))\right]^{+}\right] \\
& =\left[\left[\left(\widehat{\eta}^{c} \circ f\right)(A)\right]^{-},\left[\left(\widehat{\eta}^{c} \circ f\right)(A)\right]^{+}\right] \\
& =\left[\left[\left(f^{-1}\left[\widehat{\eta}^{c}\right]\right)(A)\right]^{-},\left[\left(f^{-1}\left[\widehat{\eta}^{c}\right]\right)(A)\right]^{+}\right] \\
& =f^{-1}\left[\widehat{\eta}^{c}\right](A) .
\end{aligned}
$$

Hence, $\left(f^{-1}[\widehat{\eta}]\right)^{c}=f^{-1}\left[\widehat{\eta}^{c}\right]$.

ii. Let $\varnothing \neq B \in f(\mathcal{I}(X))$ and $S=\{A \in \mathcal{I}(X): f(A)=B\}$. Then

$$
\begin{aligned}
(f[\widehat{\iota}])^{c}(B) & =\left[\inf _{y \in B}\left\{1-[f[\widehat{\iota}](\{y\})]^{-}\right\}, \inf _{y \in B}\left\{1-\left[f[\widehat{\iota}(\{y\})]^{+}\right\}\right]\right. \\
& \left.=\left[\inf _{y \in B}\left\{1-\sup _{A \in S^{\prime}}[\widehat{\iota}(A)]^{-}\right\}, \inf _{y \in B}\left\{1-\sup _{A \in S^{\prime}} \widehat{\iota}(A)\right]^{+}\right\}\right] ;
\end{aligned}
$$

where $S^{\prime}=\{A \in \mathcal{I}(X): f(A)=\{y\}\}$. Since $1-\sup _{A \in S^{\prime}} \widehat{\iota}(A)=\inf _{A \in S^{\prime}}\{1-\widehat{\iota}(A)\}$, we have

$$
(f[\widehat{\iota}])^{c}(B)=\left[\inf _{y \in B}\left\{\inf _{A \in S^{\prime}}\left\{1-[\widehat{\iota}(A)]^{-}\right\}\right\}, \inf _{y \in B}\left\{\inf _{A \in S^{\prime}}\left\{1-[\widehat{\iota}(A)]^{+}\right\}\right\}\right] .
$$

Let $S_{y}^{\prime}=\{\{x\} \in \mathcal{I}(X): f(\{x\})=\{y\}\}$. Observe that since $\widehat{\iota}$ is an IVFI set, if $\{x\} \subseteq A$, then $\widehat{\iota}(A) \leq_{I} \widehat{\iota}(\{x\})$ and $1-\widehat{\iota}(\{x\}) \leq_{I} 1-\widehat{\iota}(A)$. Noting that $S_{y}^{\prime} \subseteq S^{\prime}$, we thus have

$$
\left[\inf _{A \in S^{\prime}}\left\{1-[\widehat{\iota}(A)]^{-}\right\}, \inf _{A \in S^{\prime}}\left\{1-[\widehat{\iota}(A)]^{+}\right\}\right]=\left[\inf _{A \in S_{y}^{\prime}}\left\{1-[\widehat{\iota}(A)]^{-}\right\}, \inf _{A \in S_{y}^{\prime}}\left\{1-[\widehat{\iota}(A)]^{+}\right\}\right] .
$$

Hence,

$$
(f[\widehat{\iota}])^{c}(B)=\left[\inf _{y \in B}\left\{\inf _{\{x\} \in S_{y}^{\prime}}\left\{1-[\widehat{\iota}(\{x\})]^{-}\right\}\right\}, \inf _{y \in B}\left\{\inf _{\{x\} \in S_{y}^{\prime}}\left\{1-[\widehat{\iota}(\{x\})]^{+}\right\}\right\}\right] .
$$


Consider that

$$
\begin{aligned}
{\left[\inf _{y \in B}\left\{\inf _{\{x\} \in S_{y}^{\prime}}\left\{1-[\widehat{\iota}(\{x\})]^{-}\right\}\right\}\right.} & \left.\inf _{y \in B}\left\{\inf _{\{x\} \in S_{y}^{\prime}}\left\{1-[\widehat{\iota}(\{x\})]^{+}\right\}\right\}\right] \\
= & {\left[\inf _{x \in A_{B}}\left\{1-[\widehat{\iota}(\{x\})]^{-}\right\}, \inf _{x \in A_{B}}\left\{1-[\widehat{\iota}(\{x\})]^{+}\right\}\right] } \\
= & {\left[\left[\widetilde{\iota}^{c}\left(A_{B}\right)\right]^{-},\left[\widehat{\iota}^{c}\left(A_{B}\right)\right]^{+}\right], }
\end{aligned}
$$

where $A_{B}=\left\{x:\{x\} \in S_{y}^{\prime}, y \in B\right\}$. Thus,

$$
(f[\widehat{\iota}])^{c}(B)=\left[\left[\widehat{\iota}^{c}\left(A_{B}\right)\right]^{-},\left[\widehat{\iota}^{c}\left(A_{B}\right)\right]^{+}\right] .
$$

Note that $A_{B} \in S$. Then,

$$
\begin{aligned}
(f[\widehat{\imath}])^{c}(B) & =\left[\left[\widetilde{\iota}^{c}\left(A_{B}\right)\right]^{-},\left[\widehat{\iota}^{c}\left(A_{B}\right)\right]^{+}\right] \\
& \leq{ }_{I}\left[\sup _{A \in S}\left[\widetilde{\iota}^{c}(A)\right]^{-}, \sup _{A \in S}\left[\widetilde{\iota}^{c}(A)\right]^{+}\right] \\
& =\left[\left[f\left[\widetilde{\iota}^{c}\right](B)\right]^{-},\left[f\left[\widetilde{\iota}^{c}\right](B)\right]^{+}\right] \\
& =f\left[\widehat{\iota}^{c}\right](B) .
\end{aligned}
$$

Hence, $(f[\widehat{\imath}])^{c} \leqslant f\left[\widehat{\iota}^{c}\right]$.

iii. Let $A \in f^{-1}(\mathcal{I}(Y))$. Then, $A \subseteq f^{-1}(B)$, for some $B \in \mathcal{I}(Y)$. Note that $f(A) \subseteq$ $f\left(f^{-1}(B)\right) \subseteq B \in \mathcal{I}(Y)$. Thus, $f(A) \in \mathcal{I}(Y)$. If $\widehat{\omega} \leqslant \widehat{\eta}$, then $\widehat{\omega}(f(A)) \leq_{I} \widehat{\eta}(f(A))$. Consider that

$$
\left(f^{-1}[\widehat{\omega}]\right)(A)=(\widehat{\omega} \circ f)(A)=\widehat{\omega}(f(A)) \leq_{I} \widehat{\eta}(f(A))=(\widehat{\eta} \circ f)(A)=\left(f^{-1}[\widehat{\eta}]\right)(A) .
$$

Therefore, $f^{-1}[\widehat{\omega}] \leqslant f^{-1}[\widehat{\eta}]$.

iv. Let $B \in f(\mathcal{I}(X))$ and $S=\{A \in \mathcal{I}(X): f(A)=B\}$. If $\widehat{\iota} \leqslant \widehat{\tau}$, then $\widehat{\iota}(A) \leq_{I} \widehat{\tau}(A)$, for all $A \in \mathcal{I}(X)$. Hence,

$$
(f[\widehat{\iota}])(B)=\left[\sup _{A \in S}[\widehat{\iota}(A)]^{-}, \sup _{A \in S}[\widehat{\iota}(A)]^{+}\right] \leq_{I}\left[\sup _{A \in S}[\widehat{\tau}(A)]^{-}, \sup _{A \in S}[\widehat{\tau}(A)]^{+}\right]=(f[\widehat{\tau}])(B) .
$$

Therefore, $f[\widehat{\iota}] \leqslant f[\widehat{\tau}]$.

v. Suppose that $f$ is onto. Let $B \in f\left(f^{-1}(\mathcal{I}(Y))\right)=\mathcal{I}(Y)$ and $S=\left\{A \in f^{-1}(\mathcal{I}(Y))\right.$ : $f(A)=B\}$. Then

$$
\begin{aligned}
f\left[f^{-1}[\widehat{\eta}]\right](B) & =\left[\sup _{A \in S}\left[\left(f^{-1}[\widehat{\eta}]\right)(A)\right]^{-}, \sup _{A \in S}\left[\left(f^{-1}[\widehat{\eta}]\right)(A)\right]^{+}\right] \\
& =\left[\sup _{A \in S}[(\widehat{\eta} \circ f)(A)]^{-}, \sup _{A \in S}[(\widehat{\eta} \circ f)(A)]^{+}\right] \\
& =\left[\sup _{A \in S}[\widehat{\eta}(f(A))]^{-}, \sup _{A \in S}[\widehat{\eta}(f(A))]^{+}\right]
\end{aligned}
$$




$$
\begin{aligned}
& =\left[[\widehat{\eta}(B)]^{-},[\widehat{\eta}(B)]^{+}\right] \\
& =\widehat{\eta}(B) .
\end{aligned}
$$

Thus, $f\left[f^{-1}[\widehat{\eta}]\right]=\widehat{\eta}$.

vi. Suppose that $f$ is one-to-one. Let $A \in f^{-1}(f(\mathcal{I}(X)))=\mathcal{I}(X)$. Then, $A \subseteq f^{-1}(B)$, for some $B \in f(\mathcal{I}(X))$. Hence, $f(A) \subseteq B$. Since $f(\mathcal{I}(X))$ is an ideal, $f(A) \in$ $f(\mathcal{I}(X))$. Then,

$$
f^{-1}\left[f[\widehat{\iota}](A)=(f[\widehat{\iota}] \circ f)(A)=f[\widehat{\iota}](f(A))=\left[\sup _{C \in S}[\widehat{\iota}(C)]^{-}, \sup _{C \in S}[\widehat{\iota}(C)]^{+}\right],\right.
$$

where $S=\{C \in \mathcal{I}(X): f(C)=f(A)\}$. Since $A \in S$, we have

$$
\widehat{\iota}(A) \leq_{I}\left[\sup _{C \in S}[\widehat{\iota}(C)]^{-}, \sup _{C \in S}[\widehat{\iota}(C)]^{+}\right] .
$$

Thus, $\widehat{\iota} \leqslant f^{-1}[f[\widehat{\iota}]$.

The pre-image of the arbitrary union and intersection of IVFI sets is just the union and intersection of the pre-images as proved below.

Theorem 6. Let $X$ and $Y$ be nonempty sets and $\mathcal{I}(Y)$ be an ideal on $Y$. Moreover, let $f: X \rightarrow Y$ be a mapping and $\left\{\widehat{\iota}_{j}: j \in J\right\}$ be a collection of IVFI sets in $\mathscr{I}^{\mathcal{I}(Y)}$. Then

$$
\begin{aligned}
& \text { i. } f^{-1}\left[\bigvee_{j \in J} \widehat{\iota}_{j}\right]=\bigvee_{j \in J} f^{-1}\left[\widehat{\iota}_{j}\right] ; \text { and } \\
& \text { ii. } f^{-1}\left[\bigwedge_{j \in J} \widehat{\iota}_{j}\right]=\bigwedge_{j \in J} f^{-1}\left[\widehat{\iota}_{j}\right] .
\end{aligned}
$$

Proof. Let $A \in f^{-1}(\mathcal{I}(Y))$. Consider that

$$
\begin{aligned}
\left(f^{-1}\left[\bigvee_{j \in J} \widehat{\iota}_{j}\right]\right)(A) & =\left(\bigvee_{j \in J} \widehat{\iota}_{j} \circ f\right)(A) \\
& =\left(\bigvee_{j \in J} \widehat{\iota}_{j}\right)(f(A)) \\
& =\left[\sup _{j \in J}\left\{\left[\widehat{\iota}_{j}(f(A))\right]^{-}\right\}, \sup _{j \in J}\left\{\left[\widehat{\iota}_{j}(f(A)]^{+}\right\}\right]\right. \\
& =\left[\sup _{j \in J}\left\{\left[\left(\widehat{\iota}_{j} \circ f\right)(A)\right]^{-}\right\}, \sup _{j \in J}\left\{\left[\left(\widehat{\iota}_{j} \circ f\right)(A)\right]^{+}\right\}\right]
\end{aligned}
$$




$$
=\bigvee_{j \in J}\left(\widehat{\iota}_{j} \circ f\right)(A)=\left(\bigvee_{j \in J} f^{-1}\left[\widehat{\iota}_{j}\right]\right)(A) .
$$

Thus, $f^{-1}\left[\bigvee_{j \in J} \widehat{\iota}_{j}\right]=\bigvee_{j \in J} f^{-1}\left[\widehat{\iota}_{j}\right]$, proving $(i)$. Result (ii) can be proved similarly.

\section{Topology and continuity}

With the operations on IVFI sets, we can have an analogue of the classical topology.

Definition 6. Let $X$ be a nonempty set and $\mathcal{I}(X)$ be an ideal on $X$. An IVFI topology is a family $\mathscr{T}^{\prime}$ of IVFI sets such that:

(i) $\widetilde{0}_{\mathcal{I}(X)}, \widetilde{1}_{\mathcal{I}(X)} \in \mathscr{T}^{\prime}$

(ii) if $\widehat{\iota}, \widehat{\tau} \in \mathscr{T}^{\prime}$, then $\widehat{\iota} \wedge \widehat{\tau} \in \mathscr{T}^{\prime}$; and

(iii) if $\left\{\widehat{\iota}_{j}: j \in J\right\} \subseteq \mathscr{T}^{\prime}$, then $\bigvee_{j \in J} \widehat{\iota}_{j} \in \mathscr{T}^{\prime}$.

We call the ordered pair $\left(\mathcal{I}(X), \mathscr{T}^{\prime}\right)$ an IVFI space and an element of $\mathscr{T}^{\prime}$ an IVFI open set. An IVFI set $\widehat{\iota}$ will be called IVFI closed if its complement is IVFI open.

It can be easily seen that if $\left\{\mathscr{T}_{\alpha}^{\prime}: \alpha \in \mathscr{A}\right\}$ is a family of IVFI topologies on $\mathcal{I}(X)$, then $\bigcap_{\alpha \in \mathscr{A}} \mathscr{T}_{\alpha}^{\prime}$ is also an IVFI topology. However, $\bigcup_{\alpha \in \mathscr{A}} \mathscr{T}_{\alpha}^{\prime}$ need not be.

Let $\left(\mathcal{I}(X), \mathscr{T}^{\prime}\right)$ be an IVFI space. We call the subcollection $\mathcal{B}$ of $\mathscr{T}^{\prime}$ an IVFI base for $\mathscr{T}^{\prime}$ if every member of $\mathscr{T}^{\prime}$ can be expressed as a union of members of $\mathcal{B}$. Let $[0,0] \neq \alpha \in \mathscr{I}$ and $\varnothing \neq B \in \mathcal{I}(X)$. We call the IVFI set given by

$$
P_{\alpha, B}(A)=\left\{\begin{array}{cll}
\alpha & , & \text { if } A \subseteq B, A \neq \varnothing ; \\
{[0,0]} & , & \text { otherwise. }
\end{array}\right.
$$

an IVFI point. One can calculate that the explicit form of the complement of $P_{\alpha, B}$ is given by

$$
P_{\alpha, B}^{c}(A)=\left\{\begin{array}{cl}
{[0,0]} & , \quad \text { if } A=\varnothing ; \\
{\left[1-\alpha^{+}, 1-\alpha^{-}\right]} & , \quad \text { if } A \cap B \neq \varnothing ; \\
{[1,1]} & , \quad \text { if } A \neq \varnothing \text { and } A \cap B=\varnothing .
\end{array}\right.
$$

We say that $P_{\alpha, B}$ is contained in an IVFI set $\widehat{\iota}$, denoted by $P_{\alpha, B} \in \widehat{\iota}$, if and only if $\alpha \leq_{I} \widehat{\iota}(B)$. With this, we can characterize an IVFI base and IVFI open sets.

Remark 6. Every IVFI set $\widehat{\iota}$ can be expressed as the union of all IVFI points which is contained in $\widehat{\iota}$. That is, if $\widehat{\iota}(B)$ is not zero for $B \in \mathcal{I}(X)$, then

$$
\widehat{\iota}(B)=\sup \left\{\alpha: P_{\alpha, B} \text { is an IVFI point and }[0,0]<_{I} \alpha \leq_{I} \widehat{\iota}(B)\right\} \text {. }
$$


Theorem 7. Let $\left(\mathcal{I}(X), \mathscr{T}^{\prime}\right)$ be an IVFI space and $\mathcal{B} \subseteq \mathscr{T}^{\prime}$. Then $\mathcal{B}$ is an IVFI base for $\left(\mathcal{I}(X), \mathscr{T}^{\prime}\right)$ if and only if for each $\widehat{\iota} \in \mathscr{T}^{\prime}$ and for each IVFI point $P_{\alpha, B} \in \widehat{\iota}$ where $[0,0] \neq \alpha \in \mathscr{I}$ and $\varnothing \neq B \in \mathcal{I}(X)$, there exists $\widehat{\rho} \in \mathcal{B}$ such that $P_{\alpha, B} \in \widehat{\rho} \leqslant \widehat{\iota}$.

Proof. Suppose that $\mathcal{B}$ is an IVFI base for $\left(\mathcal{I}(X), \mathscr{T}^{\prime}\right)$. Let $\widehat{\iota} \in \mathscr{T}^{\prime}$ and $P_{\alpha, B} \in \widehat{\iota}$, where $[0,0] \neq \alpha \in \mathscr{I}$ and $\varnothing \neq B \in \mathcal{I}(X)$. Then by the definition of an IVFI base, there exists $\mathcal{C} \subseteq \mathcal{B}$ such that $\widehat{\iota}=\bigvee_{\widehat{\rho} \in \mathcal{C}} \widehat{\rho}$. Since $P_{\alpha, B} \in \widehat{\iota}, P_{\alpha, B} \in \widehat{\rho}$ for some $\widehat{\rho} \in \mathcal{C}$. Consequently, $P_{\alpha, B} \in \widehat{\rho} \leqslant \widehat{\iota}$.

Conversely, suppose that for each $\widehat{\iota} \in \mathscr{T}^{\prime}$ and for each IVFI point $P_{\alpha, B} \in \widehat{\iota}$ where $[0,0] \neq \alpha \in \mathscr{I}$ and $\varnothing \neq B \in \mathcal{I}(X)$, there exists $\widehat{\rho}_{\alpha, B} \in \mathcal{B}$ such that $P_{\alpha, B} \in \widehat{\rho}_{\alpha, B} \leqslant \widehat{\iota}$. Let $\widehat{\iota} \in \mathscr{T}^{\prime}$ and consider any arbitrary $P_{\alpha, B} \in \widehat{\iota}$. By Remark 6, we have $\widehat{\iota}=\bigvee_{P_{\alpha, B} \in \hat{\iota}} P_{\alpha, B}$. Since $P_{\alpha, B} \in \widehat{\rho}_{\alpha, B}$, it follows that $\bigvee P_{\alpha, B} \leqslant \bigvee \widehat{\rho}_{\alpha, B}$, and so $\widehat{\iota} \leqslant \bigvee \widehat{\rho}_{\alpha, B}$. But note $P_{\alpha, B} \in \hat{\iota} \quad P_{\alpha, B} \in \hat{\iota} \quad P_{\alpha, B} \in \hat{\imath}$

that $\widehat{\rho}_{\alpha, B} \leqslant \widehat{\iota}$. Thus, we have $\bigvee_{P_{\alpha, B} \in \widehat{\iota}} \widehat{\rho}_{\alpha, B} \leqslant \bigvee_{P_{\alpha, B} \in \widehat{\iota}} \widehat{\iota}=\widehat{\iota}$. Hence, $\widehat{\iota}=\bigvee_{P_{\alpha, B} \in \widehat{\iota}} \widehat{\rho}_{\alpha, B}$. Therefore, $\mathcal{B}$ is an IVFI base for $\mathscr{T}^{\prime}$.

Corollary 3. Let $\left(\mathcal{I}(X), \mathscr{T}^{\prime}\right)$ be an IVFI space and $\mathcal{B}$ an IVFI base. Then, $\widehat{\imath} \in \mathscr{T}^{\prime}$ if and only if for each $P_{\alpha, B} \in \widehat{\iota}$ where $[0,0] \neq \alpha \in \mathscr{I}$ and $\varnothing \neq B \in \mathcal{I}(X)$, there exists $\widehat{\omega} \in \mathcal{B}$ such that $P_{\alpha, B} \in \widehat{\omega} \leqslant \widehat{\iota}$.

Proof. Let $\widehat{\iota} \in \mathscr{T}^{\prime}$ and $P_{\alpha, B} \in \widehat{\iota}$, where $[0,0] \neq \alpha \in \mathscr{I}$ and $\varnothing \neq B \in \mathcal{I}(X)$. Then by Theorem 7 , there exists $\widehat{\omega} \in \mathcal{B}$ such that $P_{\alpha, B} \in \widehat{\omega} \leqslant \widehat{\iota}$.

Conversely, suppose that for each $P_{\alpha, B} \in \widehat{\iota}$ where $[0,0] \neq \alpha \in \mathscr{I}$ and $\varnothing \neq B \in \mathcal{I}(X)$, there exists $\widehat{\omega}_{\alpha, B} \in \mathcal{B}$ such that $P_{\alpha, B} \in \widehat{\omega}_{\alpha, B} \leqslant \widehat{\iota}$. Then by Theorem $7, \widehat{\iota}=\bigvee_{P_{\alpha, B} \in \widehat{\iota}} \widehat{\omega}_{\alpha, B}$ where $\widehat{\omega}_{\alpha, B} \in \mathcal{B}$. Since $\mathcal{B}$ is an IVFI base, we should have $\widehat{\iota}=\bigvee_{P_{\alpha, B} \in \widehat{\iota}} \widehat{\omega}_{\alpha, B} \in \mathscr{T}^{\prime}$.

Let $\hat{\imath}$ be an IVFI set in an IVFI space $\left(\mathcal{I}(X), \mathscr{T}^{\prime}\right)$. We define the IVFI interior of $\widehat{\iota}$, denoted by int $\widehat{\iota}$, by int $\widehat{\iota}=\bigvee\left\{\widehat{\tau}: \widehat{\tau} \leqslant \widehat{\iota}, \widehat{\tau} \in \mathscr{T}^{\prime}\right\}$, and the IVFI closure of $\widehat{\iota}$, denoted by $c l \widehat{\iota}$, by $c l \widehat{\iota}=\bigwedge\left\{\widehat{\omega}: \widehat{\iota} \leqslant \widehat{\omega}, \widehat{\omega}^{c} \in \mathscr{T}^{\prime}\right\}$. One can show, that indeed just like the usual topology, the IVFI interior is the largest IVFI open set contained in $\widehat{\iota}$ and the IVFI closure is the smallest IVFI closed set containing $\widehat{\iota}$. Moreover, $\widehat{\iota}$ is IVFI open if and only if $\widehat{\iota}=$ int $\widehat{\iota}$ and $\widehat{\iota}$ is IVFI closed if and only if $\widehat{\iota}=c l \widehat{\iota}$.

The importance of the following concept will be seen when dealing with continuity with respect to IVFI sets.

Definition 7. Let $\left(\mathcal{I}(X), \mathscr{T}^{\prime}\right)$ be an IVFI space. An IVFI set $\widehat{\eta}$ is said to be an IVFI neighborhood of an IVFI point $P_{\alpha, B}$, where $[0,0] \neq \alpha \in \mathscr{I}$ and $\varnothing \neq B \in \mathcal{I}(X)$, if there exists $\widehat{\omega} \in \mathscr{T}^{\prime}$ such that $P_{\alpha, B} \in \widehat{\omega} \leqslant \widehat{\eta}$. An IVFI neighborhood $\widehat{\eta}$ of an IVFI point $P_{\alpha, B}$ is said to be an IVFI open neighborhood if $\widehat{\eta} \in \mathscr{T}^{\prime}$. 
Theorem 8. Let $\left(\mathcal{I}(X), \mathscr{T}^{\prime}\right)$ be an IVFI space. Then $\widehat{\eta} \in \mathscr{T}^{\prime}$ if and only if for every IVFI point $P_{\alpha, B} \in \widehat{\eta}$ where $[0,0] \neq \alpha \in \mathscr{I}$ and $\varnothing \neq B \in \mathcal{I}(X), \widehat{\eta}$ is an IVFI neighborhood of $P_{\alpha, B}$.

Proof. Let $\widehat{\eta} \in \mathscr{T}^{\prime}$ and $P_{\alpha, B} \in \widehat{\eta}$ be an IVFI point where $[0,0] \neq \alpha \in \mathscr{I}$ and $\varnothing \neq B \in \mathcal{I}(X)$. Then by Theorem 3 , there exists $\widehat{\omega} \in \mathcal{B}$ where $\mathcal{B}$ is an IVFI base for $\mathscr{T}^{\prime}$ such that $P_{\alpha, B} \in \widehat{\omega} \leqslant \widehat{\eta}$. But note that every members of $\mathcal{B}$ are basic IVFI open sets of $\mathscr{T}^{\prime}$, and so $\widehat{\omega} \in \mathscr{T}^{\prime}$. Thus, there exists $\widehat{\omega} \in \mathscr{T}^{\prime}$ such that $P_{\alpha, B} \in \widehat{\omega} \leqslant \widehat{\eta}$. Hence, $\widehat{\eta}$ is an IVFI neighborhood of $P_{\alpha, B}$.

Conversely, suppose that for every IVFI point $P_{\alpha, B} \in \widehat{\eta}$ where $[0,0] \neq \alpha \in \mathscr{I}$ and $\varnothing \neq B \in \mathcal{I}(X), \widehat{\eta}$ is an IVFI neighborhood of $P_{\alpha, B}$. Then there exists $\widehat{\omega}_{\alpha, B} \in \mathscr{T}^{\prime}$ such that $P_{\alpha, B} \in \widehat{\omega}_{P_{\alpha, B}} \leqslant \widehat{\eta}$. By Theorem $7, \widehat{\eta}=\bigvee_{P_{\alpha, B} \in \widehat{\eta}} \widehat{\omega}_{\alpha, B}$. Since each $\widehat{\omega}_{\alpha, B} \in \mathscr{T}^{\prime}$, we have $\bigvee \widehat{\omega}_{P_{\alpha, B}} \in \mathscr{T}^{\prime}$. Hence, $\widehat{\eta} \in \mathscr{T}^{\prime}$. $P_{\alpha, B} \in \widehat{\eta}$

Due to Theorem 2, we have the following result which is an instance of the difference of the IVFI topology from the usual topology.

Theorem 9. Let $\widehat{\iota}$ be an IVFI set in an IVFI space $\left(\mathcal{I}(X), \mathscr{T}^{\prime}\right)$. Then, int $\widehat{\iota} \leqslant\left(c l \widehat{\iota}^{c}\right)^{c}$ and $c l \hat{\iota} \leqslant\left(\text { int } \widehat{\iota}^{c}\right)^{c}$.

Proof. Let $\widehat{\iota}$ be an IVFI set in an IVFI space $\left(\mathcal{I}(X), \mathscr{T}^{\prime}\right)$. Then

$$
\begin{aligned}
\text { int } \widehat{\iota} & =\bigvee\left\{\widehat{\tau}: \widehat{\tau} \leqslant \widehat{\iota}, \widehat{\tau} \in \mathscr{T}^{\prime}\right\} \\
& \leqslant \bigvee\left\{\left(\widehat{\tau}^{c}\right)^{c}:\left(\widehat{\tau}^{c}\right)^{c} \leqslant\left(\widehat{\iota}^{c}\right)^{c}, \widehat{\tau} \in \mathscr{T}^{\prime}\right\} \\
& =\left(\bigwedge\left\{\widehat{\tau}^{c}: \widehat{\iota}^{c} \leqslant \widehat{\tau}^{c}, \widehat{\tau} \in \mathscr{T}^{\prime}\right\}\right)^{c} \\
& =\left(c l \widehat{\iota}^{c}\right)^{c}
\end{aligned}
$$

and

$$
\begin{aligned}
c l \widehat{\iota} & =\bigwedge\left\{\widehat{\omega}: \widehat{\iota} \leqslant \widehat{\omega}, \widehat{\omega}^{c} \in \mathscr{T}^{\prime}\right\} \\
& \leqslant \bigwedge\left\{\left(\widehat{\omega}^{c}\right)^{c}:\left(\widehat{\iota}^{c}\right)^{c} \leqslant\left(\widehat{\omega}^{c}\right)^{c}, \widehat{\omega}^{c} \in \mathscr{T}^{\prime}\right\} \\
& =\left(\bigvee\left\{\widehat{\omega}^{c}: \widehat{\omega}^{c} \leqslant \widehat{\iota}^{c}, \widehat{\omega}^{c} \in \mathscr{T}^{\prime}\right\}\right)^{c} \\
& =\left(\text { int } \widehat{\iota}^{c}\right)^{c} .
\end{aligned}
$$

We next make precise what we mean by continuity with respect to IVFI sets. Let $f: X \rightarrow Y$ be a one-to-one map and $\mathcal{I}(X)$ be an ideal on $X$. Then, $f(\mathcal{I}(X))$ is an ideal on $Y$, by Theorem 3 , and by Proposition $2, f^{-1}(f(\mathcal{I}(X)))=\mathcal{I}(X)$.

Definition 8. Let $f: X \rightarrow Y$ be a one-to-one map and $\mathcal{I}(X)$ be an ideal on $X$. Let $\mathscr{T}_{1}^{\prime}$ and $\mathscr{T}_{2}^{\prime}$ be IVFI topologies on $\mathcal{I}(X)$ and $f(\mathcal{I}(X))$, respectively. The map $f$ is said to be IVFI continuous if $f^{-1}[\hat{\imath}] \in \mathscr{T}_{1}^{\prime}$, for all $\widehat{\iota} \in \mathscr{T}_{2}^{\prime}$. 
The next theorem characterizes the IVFI continuous maps.

Theorem 10. Let $f: X \rightarrow Y$ be a one-to-one map and $\mathcal{I}(X)$ be an ideal on $X$. Let $\mathcal{I}(Y)=f(\mathcal{I}(X))$. Moreover, let $\left(\mathcal{I}(X), \mathscr{T}_{1}^{\prime}\right)$ and $\left(\mathcal{I}(Y), \mathscr{T}_{2}^{\prime}\right)$ be IVFI spaces of $\mathcal{I}(X)$ and $\mathcal{I}(Y)$, respectively. Then the following statements are equivalent:

(i) the function $f$ is IVFI continuous;

(ii) the inverse image of every IVFI closed set is IVFI closed;

(iii) for each IVFI point $P_{\alpha, B}$, the inverse of every neighborhood of $f\left[P_{\alpha, B}\right]$ under $f$ is neighborhood of $P_{\alpha, B}$;

(iv) for each IVFI point $P_{\alpha, B}$ and each neighborhood $\widehat{\eta}$ of $f\left[P_{\alpha, B}\right]$, there is a neighborhood $\widehat{\eta}^{\prime}$ of $P_{\alpha, B}$ such that $f\left[\widehat{\eta}^{\prime}\right]=\widehat{\eta}$ whenever $f$ is onto;

(v) $f[c l \hat{\iota}] \leqslant c l f[\widehat{\iota}]$ for all $\widehat{\iota} \in \mathscr{I}^{\mathcal{I}(X)}$; and

(vi) $c l f^{-1}[\widehat{\omega}] \leqslant f^{-1}[$ cl $\widehat{\omega}]$ for all $\widehat{\omega} \in \mathscr{I}^{\mathcal{I}(Y)}$, whenever $f$ is onto.

Proof. $(i) \Longleftrightarrow($ ii $)$

Suppose that $f$ is IVFI continuous. Let $\widehat{\iota}$ be an IVFI closed set in $\mathscr{I}^{\mathcal{I}(Y)}$. Then $\widehat{\iota}^{c} \in \mathscr{T}_{2}^{\prime}$. Since $f$ is IVFI continuous, $f^{-1}\left[\widehat{\iota}^{c}\right] \in \mathscr{T}_{1}^{\prime}$. Note that by Theorem $5(i), f^{-1}\left[\hat{\iota}^{c}\right]=\left(f^{-1}[\hat{\imath}]\right)^{c}$. Thus, $f^{-1}[\tilde{\iota}]$ is an IVFI closed set in $\mathscr{I}^{\mathcal{I}(X)}$.

Conversely, suppose that the inverse image of every IVFI closed set is IVFI closed. Let $\widehat{\tau} \in \mathscr{T}_{2}^{\prime}$. Then $\widehat{\tau}^{c}$ is an IVFI closed set in $\mathscr{I}^{\mathcal{I}(Y)}$. By assumption, $f^{-1}\left[\widehat{\tau}^{c}\right]$ is an IVFI closed set in $\mathscr{I}^{\mathcal{I}(X)}$. Note that by Theorem $5(i),\left(f^{-1}[\widehat{\tau}]\right)^{c}=f^{-1}\left[\widehat{\tau}^{c}\right]$. Thus, $f^{-1}[\widehat{\tau}] \in \mathscr{T}_{1}^{\prime}$. Hence, $f$ is IVFI continuous.

$(i i) \Longleftrightarrow(v i)$

Suppose that the inverse image of every IVFI closed set is IVFI closed. Let $\widehat{\omega}$ be an IVFI closed set in $\mathscr{I}^{\mathcal{I}(Y)}$. Then by assumption, $f^{-1}[\widehat{\omega}]$ is an IVFI closed set in $\mathscr{I}^{\mathcal{I}(X)}$. Thus, $c l f^{-1}[\widehat{\omega}]=f^{-1}[\widehat{\omega}]$. But $\widehat{\omega} \leqslant c l \widehat{\omega}$ for all $\widehat{\omega} \in \mathscr{I}^{\mathcal{I}(Y)}$, and so $f^{-1}[\widehat{\omega}] \leqslant f^{-1}[c l \widehat{\omega}]$. Hence, $c l f^{-1}[\widehat{\omega}] \leqslant f^{-1}[c l \widehat{\omega}]$ for all $\widehat{\omega} \in \mathscr{I}^{\mathcal{I}(Y)}$.

Conversely, suppose that $c l f^{-1}[\widehat{\omega}] \leqslant f^{-1}[c l \widehat{\omega}]$ for all $\widehat{\omega} \in \mathscr{I}^{\mathcal{I}(Y)}$ whenever $f$ is onto. Let $\widehat{\omega}$ be an IVFI closed set in $\mathscr{I}^{\mathcal{I}(Y)}$. Then by assumption, $c l f^{-1}[\widehat{\omega}] \leqslant f^{-1}[c l \widehat{\omega}]$. Since $\widehat{\omega}$ be an IVFI closed set in $\mathscr{I}^{\mathcal{I}(Y)}$, cl $\widehat{\omega}=\widehat{\omega}$, and so $f^{-1}[c l \widehat{\omega}]=f^{-1}[\widehat{\omega}]$. But $f^{-1}[\widehat{\omega}] \leqslant c l f^{-1}[\widehat{\omega}]$, and so $c l f^{-1}[\widehat{\omega}] \leqslant f^{-1}[\widehat{\omega}] \leqslant c l f^{-1}[\widehat{\omega}]$. Thus, $c l f^{-1}[\widehat{\omega}]=f^{-1}[\widehat{\omega}]$. Hence, $f^{-1}[\widehat{\omega}]$ is an IVFI closed set in $\mathscr{I}^{\mathcal{I}(X)}$.

$(v i) \Longleftrightarrow(v)$

Suppose that $c l f^{-1}[\widehat{\omega}] \leqslant f^{-1}[c l \widehat{\omega}]$ for all $\widehat{\omega} \in \mathscr{I}^{\mathcal{I}(Y)}$ whenever $f$ is onto. Let $\widehat{\iota} \in$ $\mathscr{I}^{\mathcal{I}(X)}$ and put $\widehat{\omega}=f[\widehat{\iota}] \in \mathscr{I}^{\mathcal{I}(Y)}$. Then by assumption, $c l f^{-1}[\widehat{\omega}] \leqslant f^{-1}[c l \widehat{\omega}]$, that is $c l f^{-1}[f[\widehat{\iota}]] \leqslant f^{-1}[\operatorname{cl} f[\widehat{\iota}]]$. Note that by Theorem $5(v i), \widehat{\iota} \leqslant f^{-1}[f[\widehat{\imath}]]$, and so $c l \widehat{\iota} \leqslant$ $c l f^{-1}[f[\hat{\imath}]]$. Thus, $c l \hat{\imath} \leqslant f^{-1}[\operatorname{cl} f[\hat{\imath}]]$. Taking the images, we have $f[c l \widehat{\iota}] \leqslant f\left[f^{-1}[\operatorname{cl} f[\widehat{\imath}]]\right]$. Since $f$ is onto, by Theorem $5(v), f\left[f^{-1}[c l f[\hat{\imath}]]\right]=c l f[\widehat{\iota}]$. Hence, $f[c l \hat{\imath}] \leqslant c l f[\hat{\iota}]$.

Conversely, suppose that $f[c l \hat{\iota}] \leqslant c l f[\hat{\iota}]$ for all $\widehat{\iota} \in \mathscr{I}^{\mathcal{I}(X)}$. Let $\widehat{\iota} \in \mathscr{I}^{\mathcal{I}(Y)}$ and put $\widehat{\iota}=f^{-1}[\widehat{\omega}] \in \mathscr{I}^{\mathcal{I}(X)}$. Then by assumption, $f[c l \widehat{\iota}] \leqslant c l f[\widehat{\iota}]$, that is $f\left[c l f^{-1}[\widehat{\omega}]\right] \leqslant$ 
cl $f\left[f^{-1}[\widehat{\omega}]\right]$. Let $f$ be an onto function. Note that by Theorem $5(v), f\left[f^{-1}[\widehat{\omega}]\right]=\widehat{\omega}$, and so $c l f\left[f^{-1}[\widehat{\omega}]\right]=c l \widehat{\omega}$. Thus, $f\left[c l f^{-1}[\widehat{\omega}]\right] \leqslant c l \widehat{\omega}$. Taking the inverse images, we have $f^{-1}\left[f\left[c l f^{-1}[\widehat{\omega}]\right]\right] \leqslant f^{-1}[c l \widehat{\omega}]$. By Theorem $5(v i)$, we have $c l f^{-1}[\widehat{\omega}] \leqslant f^{-1}\left[f\left[c l f^{-1}[\widehat{\omega}]\right]\right]$. Thus, $c l f^{-1}[\widehat{\omega}] \leqslant f^{-1}[$ cl $\widehat{\omega}]$.

(iii) $\Longleftrightarrow(i v)$

Suppose that for each IVFI point $P_{\alpha, B}$, the inverse of every neighborhood of $f\left[P_{\alpha, B}\right]$ under $f$ is a neighborhood of $P_{\alpha, B}$. Let $P_{\alpha, B}$ be an IVFI point in $\mathscr{I}^{\mathcal{I}(X)}$ and $\widehat{\eta}$ be a neighborhood of $f\left[P_{\alpha, B}\right]$. Then by assumption, there is a neighborhood $f^{-1}[\widehat{\eta}]$ of $P_{\alpha, B}$. Take $\widehat{\eta}^{\prime}=f^{-1}[\widehat{\eta}]$. Thus, $f\left[\widehat{\eta}^{\prime}\right]=f\left[f^{-1}[\widehat{\eta}]\right]=\widehat{\eta}$ whenever $f$ is onto.

Conversely, suppose that for each IVFI point $P_{\alpha, B}$ and each neighborhood $\widehat{\eta}$ of $f\left[P_{\alpha, B}\right]$, there is a neighborhood $\widehat{\eta}^{\prime}$ of $P_{\alpha, B}$ such that $f\left[\widehat{\eta}^{\prime}\right]=\widehat{\eta}$ whenever $f$ is onto. Let $P_{\alpha, B}$ be an IVFI point in $\mathscr{I}^{\mathcal{I}(X)}$ and $\widehat{\eta}$ be a neighborhood of $f\left[P_{\alpha, B}\right]$. Then by assumption, there is a neighborhood $\widehat{\eta}^{\prime}$ of $P_{\alpha, B}$ such that $f\left[\widehat{\eta}^{\prime}\right]=\widehat{\eta}$. But note that by Theorem $5(v)$, $f\left[f^{-1}[\hat{\eta}]\right]=\widehat{\eta}$ whenever $f$ is onto. Take $\widehat{\eta}^{\prime}=f^{-1}[\widehat{\eta}]$ so that $f^{-1}[\widehat{\eta}]$ is a neighborhood of $P_{\alpha, B}$.

$(i v) \Longleftrightarrow(i)$

Suppose that for each IVFI point $P_{\alpha, B}$ and each neighborhood $\widehat{\eta}$ of $f\left[P_{\alpha, B}\right]$, there is a neighborhood $\widehat{\eta}^{\prime}$ of $P_{\alpha, B}$ such that $f\left[\widehat{\eta}^{\prime}\right]=\widehat{\eta}$ whenever $f$ is onto. Let $\widehat{\eta} \in \mathscr{T}_{2}^{\prime}$ and $P_{\alpha, B}$ be an IVFI point in $\mathscr{I}^{\mathcal{I}(X)}$. By assumption, $\widehat{\eta}$ is a neighborhood of $f\left[P_{\alpha, B}\right]$. Thus, there exists a neighborhood $\widehat{\eta}^{\prime}$ of $P_{\alpha, B}$ such that $f\left[\widehat{\eta}^{\prime}\right]=\widehat{\eta}$. Taking the inverse images, $f^{-1}\left[f\left[\widehat{\eta}^{\prime}\right]\right]=f^{-1}[\widehat{\eta}]$. Note that by Theorem $5(v i), \widehat{\eta}^{\prime} \leqslant f^{-1}\left[f\left[\widehat{\eta}^{\prime}\right]\right]$, and so $\widehat{\eta}^{\prime}=f^{-1}[\widehat{\eta}]$. Thus, by Theorem $8, f^{-1}[\widehat{\eta}] \in \mathscr{T}_{1}^{\prime}$. Hence, $f$ is IVFI continuous.

Conversely, suppose that $f$ is IVFI continuous. Let $P_{\alpha, B}$ be an IVFI point in $\mathscr{I}^{\mathcal{I}(X)}$ and $\widehat{\eta}$ be a neighborhood of $f\left[P_{\alpha, B}\right]$. Then there exists $\widehat{\omega} \in \mathscr{T}_{2}^{\prime}$ such that $f\left[P_{\alpha, A}\right] \in \widehat{\omega} \leqslant \widehat{\eta}$. Taking the inverse images, we have $f^{-1}\left[f\left[P_{\alpha, B}\right]\right] \in f^{-1}[\widehat{\omega}] \leqslant f^{-1}[\widehat{\eta}]$. Note that by Theorem $5(v i), P_{\alpha, B} \leqslant f^{-1}\left[f\left[P_{\alpha, B}\right]\right]$, and so $P_{\alpha, B} \in f^{-1}[\widehat{\omega}] \leqslant f^{-1}[\widehat{\eta}]$. Since $f$ is continuous, $f^{-1}[\widehat{\omega}] \in \mathscr{T}_{1}^{\prime}$. Hence, $f^{-1}[\widehat{\eta}]$ is a neighborhood of $P_{\alpha, B}$. Take $\widehat{\eta}^{\prime}=f^{-1}[\widehat{\eta}]$. Taking the images, we have $f\left[\widehat{\eta}^{\prime}\right]=f\left[f^{-1}[\widehat{\eta}]\right]=\widehat{\eta}$, whenever $f$ is onto. Hence, $f\left[\widehat{\eta}^{\prime}\right]=\widehat{\eta}$.

\section{Acknowledgements}

The first author was supported by the Department of Science and Technology (DOST) of the Philippines and the second author by the Premier Research Institute of Science and Mathematics (PRISM) of the Mindanao State University-Iligan Institute of Technology (MSU-IIT).

\section{References}

[1] D Dubois and H Prade (eds.). Fundamentals of Fuzzy Sets. Springer Science and Bussiness Media, 2012.

[2] I Grattan-Guiness. Fuzzy membership mapped onto interval and many-valued quantities. Z. Math. Logik. Grundladen Math., 22:149-160, 1975. 
[3] KU Jahn. Intervall-wertige Mengen. Math.Nach. Southern Economic Journal, 68:115$132,1975$.

[4] D Klaua. ber einen ansatz zur mehrwertigen mengenlehre. Monatsberichte der Kniglichen Preussische Akademie des Wissenschaften zu Berlin., 7:859-876, 1965.

[5] K Kuratowski. Topology. New York: Academic Press, 1966.

[6] L Mernilo-Tutanes and R Caga-anan. Fuzzy on ideal sets and a fuzzy on ideal hahnbanach theorem. Science Diliman., 30(2):70-86, 2018.

[7] R Sambuc. Functions $\phi$-floues. Application laide au diagnostic en pathologie thyroidienne. $\mathrm{PhD}$ thesis, $\mathrm{PhD}$, Université de Marseille, France, 1975.

[8] R Vaidyanathaswamy. The localisation theory in set-topology. In Proceedings of the Indian Academy of Sciences-Section A; Springer India., volume 20(1), pages 51-61, 1944.

[9] LA Zadeh. Fuzzy sets. Information Control., 8:338-353, 1965.

[10] LA Zadeh. The concept of a linguistic variable and its application to approximate reasoning i. Information Sciences., 8:199-249, 1975.

[11] LA Zadeh. Fuzzy sets as a basis for a theory of possibility. Fuzzy Sets and Systems., 1:3-28, 1978. 